\title{
Magnetization Process of Single Molecule Magnets at Low Temperatures
}

\author{
Julio F. Fernández ${ }^{1}$ and Juan J. Alonso ${ }^{2}$ \\ ${ }^{1}$ ICMA, CSIC and Universidad de Zaragoza, 50009-Zaragoza, Spain * \\ ${ }^{2}$ Física Aplicada I, Universidad de Málaga, 29071-Málaga, Spain
}

\begin{abstract}
We show that correlations established before quenching to very low temperatures, later drive the magnetization process of systems of single molecule magnets, after a magnetic field is applied at $t=0$. We also show that in SC lattices, $m \propto \sqrt{t}$, as observed in $\mathrm{Fe}_{8}$, but only for $1+2 \log _{10}\left(h_{d} / h_{w}\right)$ time decades, where $h_{d}$ is a nearest neighbor dipolar magnetic field and a spin reversal can occur only if the field on it is within $\left(-h_{w}, h_{w}\right)$. However, the $\sqrt{t}$ behavior is not universal. For BCC and FCC lattices, $m \propto t^{p}$, but $p \simeq 0.7$. The value to which $m$ finally levels off is also given.
\end{abstract}

PACS numbers: $75.45 .+\mathrm{j}, 75.50 . \mathrm{Xx}$

Keywords: quantum tunneling, single molecule magnets, magnetic relaxation, dipolar interactions, correlations

Magnetic clusters, such as $\mathrm{Fe}_{8}$ and $\mathrm{Mn}_{12}$, that make up the core of large organometallic molecules, behave at low temperatures as large single spins. Accordingly, these molecules have come to be known as single molecule magnets (SMM's) [1]. In crystals, magnetic anisotropy energies $U$ inhibit magnetic relaxation of SMM's, which can consequently take place at very small temperatures only through magnetic quantum tunneling (MQT). Dipolar interactions play then an essential role. They can give rise, upon tunneling, to Zeeman energy changes of nearly $1 \mathrm{~K}$. This exceeds by many orders of magnitude the ground state tunnel splitting energy $\Delta$ that would follow from perturbations by higher anisotropies for $\mathrm{Fe}_{8}$ and $\mathrm{Mn}_{12}$ 2]. Energy conservation would make MQT, which has been observed experimentally [3], impossible for the vast majority of spins in the system. Hyperfine interactions between the tunneling electronic spins of interest and nuclear spins open up a fairly large tunneling window of energy $\varepsilon_{w}$ such that tunneling can occur if the Zeeman energy change $2 \varepsilon_{h}$ upon tunneling is not much larger than $\varepsilon_{w}$ [4]. More precisely, the tunneling rate $\Gamma^{\prime}$ for spins at very low temperature, is given by

$$
\Gamma^{\prime}\left(\varepsilon_{h}\right) \simeq \Gamma \eta\left(\varepsilon_{h} / \varepsilon_{w}\right)
$$

where $\Gamma$ is some rate (whose value is not important for our purposes), $\eta(x) \sim 1$ for $|x|<1, \eta(x) \sim 0$ for $x>1$, and $\varepsilon_{w} \gg \Delta$. Other theories for MQT of SMM at very low temperatures have also been proposed $[5]$. We adopt Eq. (1) here, regardeless of theory or physical mechanism behind it. We let $\eta(x)=1$ for $|x|<1$ and $\eta(x)=0$ for $x \geq 1$, and refer to $\varepsilon_{w}$ as the tunnel energy window.

The interesting early time relaxation $1-m \propto \sqrt{t}$ of an initially magnetized system has been predicted [4], observed experimentally [6], further explained [7], and widely discussed [8]. An unpredicted related phenomenon was later observed by Wernsdorfer et al. [9]: the magnetization $m$ of a system of $\mathrm{Fe}_{8}$ SMM's increases as $\sqrt{t}$, where $t$ is the time after a weak magnetic field is applied to an initially unpolarized system. Conveniently, this latter effect seemed to be independent of system shape. Interesting questions arise: is this a universal effect to be found in all MQT experiments? If not, what does it depend on? How many time decades does the $\sqrt{t}$ regime cover? What is the final steady state magnetization? No explanation or simulation that we know of has been offered. We address these questions here.

We report Monte Carlo (MC) results that reproduce the $m \propto \sqrt{t}$ behavior of initially unpolarized systems. We show that this arises from correlations that develop between spins and local magnetic dipolar fields, while cooling to low temperatures, before finally quenching to experiment. Furthermore $m(t)$ depends on the cooling protocol only through the final energy $-\varepsilon_{a}$ reached just before quenching.

The main results obtained follow. All energies and magnetic fields are given in terms of $\varepsilon_{d}$ and $h_{d}$, respectively, where $-2 \varepsilon_{d}$ is the energy of two $\mathrm{S}$ spins that lie on sites $a$ distance away, that point along the line joining their two sites, $a$ is the side of a cubic unit cell, $h_{d}=\varepsilon_{d} /\left(g \mu_{B} S\right), g$ is the gyromagnetic ratio, and $\mu_{B}$ is the Bohr magneton. We also let $\sigma$ stand for the rms value of $h$ for a disordered spin configuration [10]. After quenching and applying a field $H \lesssim 1$ at $t=0$,

$$
m(t) \simeq b \varepsilon_{a} \varepsilon_{w} H \sigma^{-3} F\left(\Gamma t, \sigma / \varepsilon_{w}, \sigma / h_{0}\right),
$$

where $b \simeq 4 \sqrt{2 / \pi}$, and

$$
\begin{array}{ll}
F \simeq \Gamma t & \text { for } \Gamma t \lesssim 1 \\
F \simeq 0.7(\Gamma t)^{p} & \text { for } 1 \lesssim \Gamma t \lesssim\left(\sigma / \epsilon_{w}\right)^{1 / p} \\
F \simeq 0.5 \sigma \varepsilon_{w}^{-1} & \text { for }\left(\sigma / \varepsilon_{w}\right)^{1 / p} \lesssim \Gamma t,
\end{array}
$$

where, $h_{0}=2(2 \pi)^{2} / 3^{5 / 2} \rho_{v}, \rho_{v}$ is the number of spin sites per unit volume, $p \simeq 0.5$, for simple cubic (SC) lattices, and $p \simeq 0.7$ for body centered cubic (BCC) and face centered cubic (FCC) lattices. These results are inferred from $\mathrm{MC}$ simulations in which the energy of the magnetic system is held constant in time as well as from arguments given below. For magnetic systems that readily exchange energy with the lattice, for which the energy is not a constant of time, results are briefly discussed in the closing remarks. We first describe the sim- 


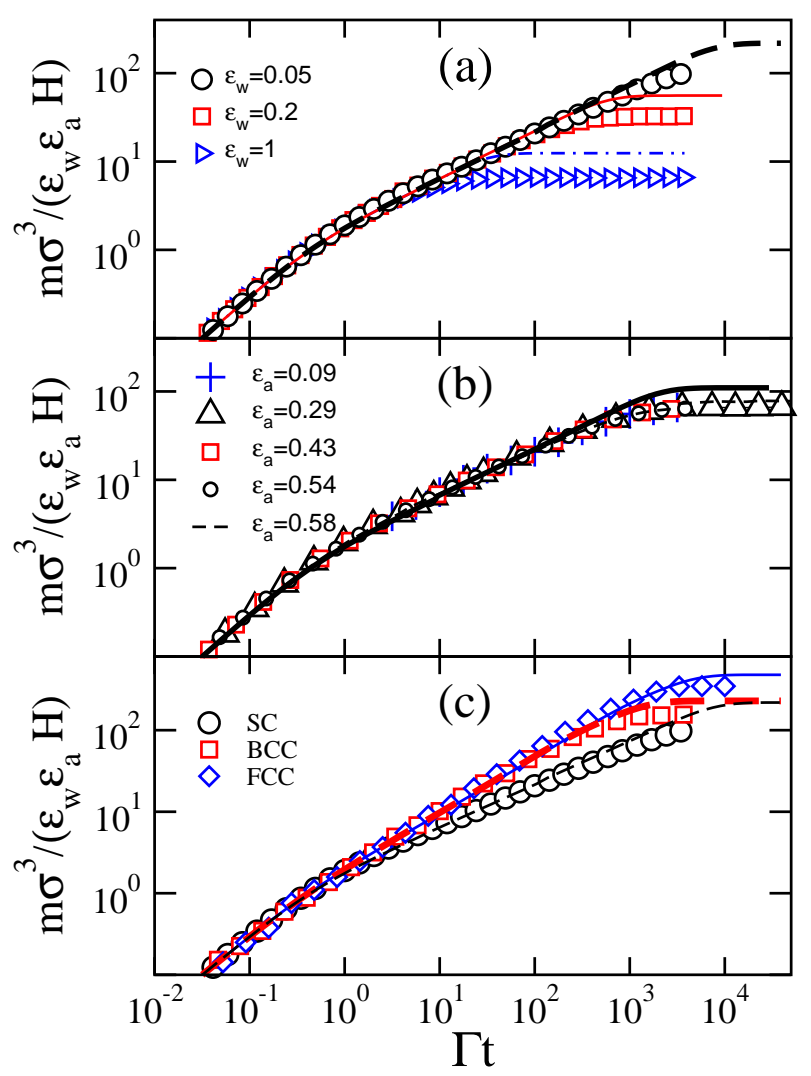

FIG. 1: (a) $m / \varepsilon_{w}$ versus $\Gamma t$ for an applied external field $H=1$ for the shown values of $\varepsilon_{w}$. First, thermalization took place from an initially disordered configuration up to the time when $\varepsilon_{a}$ reached the value 0.58 . The dashed, continuous, and dotdashed lines are for curves calculated from Eq. (11), for $\varepsilon_{w}=$ 0.05, 0.2 and 1, respectively. (b) Same as in (a) but for the shown values of $\varepsilon_{a}$ and $\varepsilon_{w}=0.1$. The continuous line, for the solution from Eq. (11). (c) Same as in (a) and (b), but for different lattices, with $\varepsilon_{w}=0.05$. For SC, BCC, and FCC lattices, previous partial thermalization took place at $T_{a}=10$, 20 and 60 , till $\varepsilon_{a}=0.31,0.28$, and 0.53 , respectively. The continuous, long dashed, and short dashed lines are for curves calculated from Eq. (11) for FCC, BCC, and SC lattices, respectively. There are no adjustable parameters.

ulations. We use the MC method to simulate magnetic relaxation of Ising systems of $\pm S$ spins, on simple cubic lattices with periodic boundary conditions, that interact through magnetic dipolar fields and flip under rules to be specified below [11]. The system is first allowed to evolve towards thermal equilibrium at some "high" temperature $T_{a}$. We assume $k_{B} T_{a} \gtrsim U / 10$, which implies that spin reversals then take place mostly through classical thermal processes. Accordingly, spin flips are then governed by detailed balance rules, and Eq. (1) is not enforced. For reasons that are given below, we also impose the restriction $T_{a} \gtrsim T_{0}$, where $T_{0}$ is the longrange ordering temperature. One may think of this first process as a waiting stage that the systems may have to undergo in the cooling process, before quenching to a lower temperature where a tunneling experiment (as in Ref. 9) can be later performed. Let this first stage end at $t=0$ with sudden cooling of the system to a "very low" temperature, that is, to a temperature below, roughly, $0.2 U /\left(S k_{B}\right)$ 3, 12. Accordingly, Eq. (1) is then enforced on all spin flips for $t>0$. As for detailed balance, we then proceed as follows. We assume that thermalization of a SMM system with the lattice does not take place (i.e., the energy is constant) at very low temperatures (but see Ref. 13]). We meet this condition by enforcing detailed balance but using an appropriately chosen pseudotemperature $T_{u}$. [From an expression below Eq. (6), $k_{B} T_{u} \approx \sigma^{2} / 2 \varepsilon_{a}$. Note that $T_{u} \geq T_{a}$, since $-\varepsilon_{a}$ cannot be smaller than the equilibrium energy at $\left.T_{a}\right]$. We have checked that the mean energy is indeed constant under this rule. We do not report here results we have obtained applying detailed balance rules with $T<T_{a}$ (applicable to systems where thermal relaxation to the lattice takes place [13]), but make a comment on them in the closing remarks. MC results for the time evolution of $m / \varepsilon_{w}$ in SC lattice systems, after a field $H=1$ is applied upon quenching, are shown in Fig. 1(a) for various values of $\varepsilon_{w}$. Before quenching, the system was thermalized at $T_{a}=10 / k_{B}$ for some time till the energy per spin reached the value -0.58 . Clearly, $m$ scales with $\varepsilon_{w}$ up to a crossover time of, roughly, $10 \Gamma^{-1} / \varepsilon_{w}^{2}$, where $m$ levels off. Within the time range $1 \lesssim \Gamma t \lesssim 10 / \varepsilon_{w}^{2}$, $m \propto \sqrt{t}$. Monte Carlo results that show how $m$ scales with $\varepsilon_{a}$ are exhibited in Fig. 1(b) for $\varepsilon_{w}=0.1$. Note also that $m_{e}$, the leveling off value of $m$, scales with $\varepsilon_{a}$, and, as argued below, it scales with $\sigma^{-2}$ as well, in agreement with Fig. 1(c). Results for different cubic lattices are shown in Fig. 1(c). The $\log m$ versus $\log t$ slopes in the intermediate time regime are clearly lattice structure dependent. Much of Eqs. (2-5) is inferred from these graphs.

For most of the rest of the paper, we try to understand these results. Let's first examine the physics of the waiting stage. We assume the system is first cooled to some temperature $T_{a}$ that is above the ordering temperature $T_{0}$, but not infinite. We also assume that $k_{B} T_{a} \gtrsim U / 10$. It then follows from Arrehnius' law, $\tau=\tau_{0} \exp \left(U / k_{B} T\right)$, that over barrier spin flipping readily takes place in the laboratory within a second's time if $\tau_{0} \lesssim 10^{-5} \mathrm{~s}$. Some correlation between spin $s_{i}$ and field $h_{i}$ at each site $i$ can therefore be established, but no long-range order can develop if $T_{a} \gtrsim T_{0}$. Assume that either $T_{a} \gg T_{0}$ or that the time spent in the waiting stage is so short that the probability density function (PDF) $p(h)$ that a randomly chosen site have field $h$ is reasonably approximated by $p(h) \propto \exp \left(-h^{2} / 2 \sigma^{2}\right)$. On the other hand, the conditional PDF to find $\pm S$ given a field $h$ acting on the spin fulfills, in equilibrium, $p( \pm S \mid h) \propto \exp \left( \pm h / k_{B} T\right)$. Now, since the joint probability density $p( \pm S, h)$ that, 
on a randomly chosen site, one find $h$ acting on $\pm S$ is in general given by $p( \pm S, h)=p( \pm S \mid h) p(h)$,

$$
p( \pm S, h) \propto e^{-\left(h \mp \sigma^{2} / k_{B} T\right)^{2} / 2 \sigma^{2}}
$$

follows in equilibrium. Therefore, the mean energy is $-\sigma^{2} / 2 k_{B} T$. The replacement $\sigma^{2} / 2 k_{B} T \rightarrow \varepsilon_{a}$, generalizes the above equation to

$$
p( \pm S, h) \propto e^{-\left(h \mp 2 \varepsilon_{a}\right)^{2} / 2 \sigma^{2}}
$$

for all times up to equilibration. Then, to first order in $\varepsilon_{a} / \sigma$

$$
p_{\uparrow}(h)-p_{\downarrow}(h) \simeq \sqrt{2 / \pi} h \varepsilon_{a} \sigma^{-3} e^{-h^{2} / 2 \sigma^{2}},
$$

where $p_{\uparrow}(h)=p(S, h)$ and $p_{\downarrow}(h)=p(-S, h)$. All points for $\left[p_{\uparrow}(h)-p_{\downarrow}(h)\right] /\left(h \varepsilon_{a}\right)$ obtained from MC calculations collapse onto a single curve in Fig. 2a, in agreement with Eq. (8). Deviations, of higher order in $\varepsilon_{a} / \sigma$, from Eq. (8) do occur. They are within $10 \%$ even if complete thermal equilibration is allowed to take place as long as $T \gtrsim 10$, i.e., above approximately $4 T_{0}$.

We now examine the time evolution of the system after abruptly cooling it, at time $t=0$, to a temperature below, roughly, $0.2 U /\left(S k_{B}\right)$. Then, spin flips up to $\left|S_{z}\right|<S$ states can be neglected, and tunneling through the ground state doublet is the only available path for spin reversals. Accordingly, spin flips are allowed only if the spin's Zeeman energy is within the tunnel window. A field $H$ is applied for all $t>0$. Now, if either the system is in thermal contact with a reservoir at a temperature such that $k_{B} T \gg \varepsilon_{w}$, or the energy is constant and sufficiently high such that $k_{B} T_{u} \gg \varepsilon_{w}$, then

$$
\dot{m}=2 \Gamma \int d h \eta(H+h) f(h, t),
$$

where $f(h, t)=p_{\downarrow}(h, t)-p_{\uparrow}(h, t)$.

It is worth pointing out that $-f(h, 0)$ is given by Eq. (8), and therefore $f(h, 0)=-\left(2 h \varepsilon_{a} / \sigma^{2}\right) p(h, 0)$ at $t=0$, where $p(h, 0)$ is the PDF regardless of spin orientation, i.e., $p(h)=p_{\downarrow}(h)+p_{\uparrow}(h)$. However, $f(h, t) \propto h p(h, t)$ does not hold for $t>0$. This point is illustrated in Fig. 2(b), where MC results for both $f(h, t)$ and $p(h, t)$ are shown for some non-zero times. $p(h, t)$ is invariant for times $\sim \Gamma$. As reported in Ref. [14], a sharp dip, of approximately $\varepsilon_{w}$ half-width, does develop in $p(h)$, but only at much later times, and then not when $k_{B} T \gg \varepsilon_{w}$. On the other hand, a hole does show up in $f(h, t)$ in Fig. 2(b), as in Wernsdorder's experiments [9], performed at $T=40 \mathrm{mK}$ (which is roughly 10 times as large as $\varepsilon_{w} / k_{B}$ ) [9, 14].

The time development of the hole in $f(h, t)$ is illustrated in Fig. 2(c). Note that the hole deepens, but its width remains approximately constant while $\Gamma t \ll 1$, and then, under the conditions stated above Eq. (9),
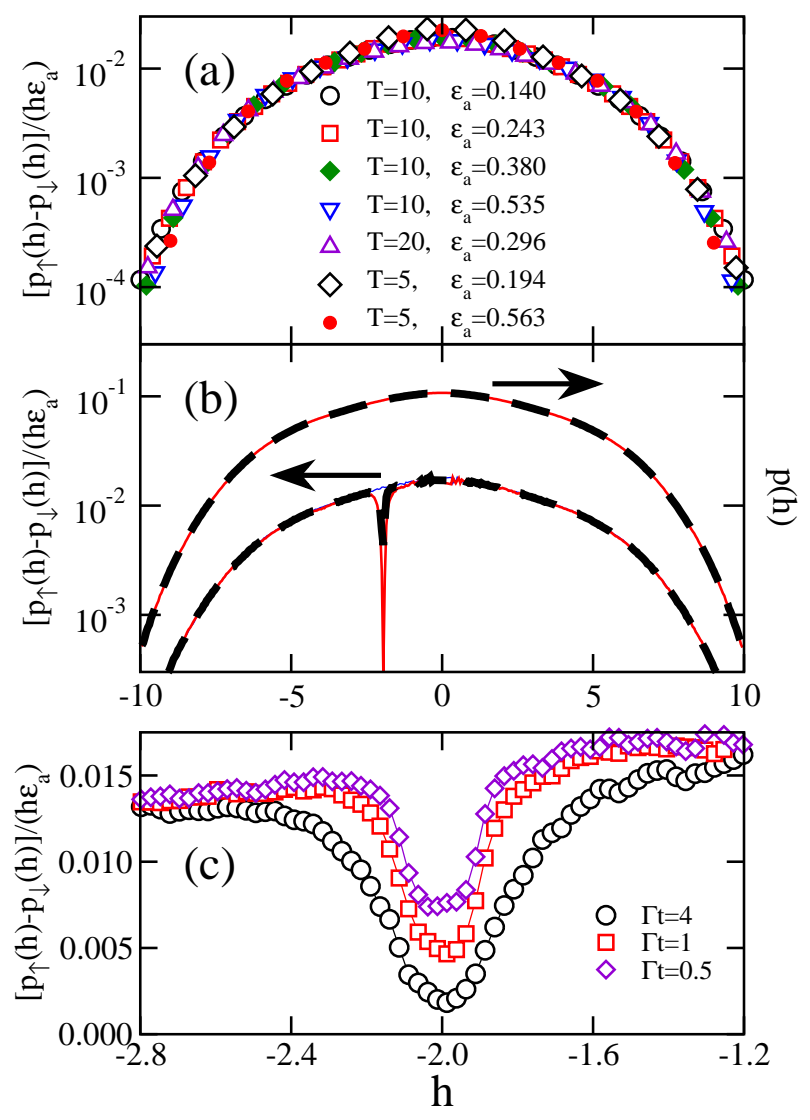

FIG. 2: $\left[p_{\uparrow}(h)-p_{\downarrow}(h)\right] /\left(h \varepsilon_{a}\right)$ for a system of $16 \times 16 \times 16$ spins at $T_{a}=10$ (recall $T_{0} \simeq 2.5$ ), before quenching. The shown temperatures are given in units of $\varepsilon_{d} / k_{B}$. The system was cooled to these "high" temperatures from an infinite temperature, and allowed to thermalize for a time, till the shown energies were reached. All points stand for averages over $1.5 \times 10^{5}$ runs. (b) $\left[p_{\downarrow}(h)-p_{\uparrow}(h)\right] /\left(h \varepsilon_{a}\right)$ and $p(h) \equiv p_{\downarrow}(h)+p_{\uparrow}(h)$ at times $t=0, \Gamma t=0.62$ (discontinuous line), and $\Gamma t=2.19$ (continuous line), for the same system as in (a), after the system was first thermalized at $T=10$ till $\varepsilon_{a} \simeq 0.25$, was then further cooled to $T=1$, and $H=2$ was then applied. A tunnel window $\varepsilon_{w}=0.1$ was enforced. All points in (b) stand for averages over $4 \times 10^{4}$ histories. (c) Same as in (b) but for $\Gamma t=0.5,1$, and 4 ; in addition, while $t<0$, the system had been partially thermalized at $T=10$ till $\varepsilon_{a}=0.247$.

$\dot{f}=-2 \Gamma f$. Therefore, since $m$ equals the area coverd by the hole [i.e., $\left.m(t)=\int d h[f(h, 0)-f(h, t)]\right]$,

$$
m \simeq 2 \varepsilon_{w} f(-H, 0)\left(1-e^{-2 \Gamma t}\right)
$$

if $\Gamma t \ll 1$ and $\varepsilon_{w} \ll \sigma$. Using Eq. (8), Eq. (3) follows. The value $m_{e}$ that $m(t)$ levels off to after a sufficiently long time, that is, Eq. (5), can be estimated as follows. For $\Gamma t \gtrsim 1, f(-H, t) \ll f(-H, 0)$, and the hole only becomes broader, but it cannot become wider than the field distribution $p(h)$. The final area covered by the hole is therefore $\sim 2 f(-H, 0) \sigma$, which is the estimated value of $m_{e}$, in rough agreement with the expression for $m_{e}$ below Eq. (5). 
More detailed considerations underlie Eq. (4). We have derived [15] the equation,

$$
\dot{x} \simeq c_{1} \sqrt{\frac{2}{\pi}}-c_{2} \int_{0}^{t} d \tau \frac{2 \varepsilon_{w} \dot{x}(\tau)}{\omega(t-\tau)+2 \varepsilon_{w}},
$$

where, $c_{1}$ and $c_{2}$ are constants to be specified below, $\omega(t-\tau)$ is the inverse of the PDF that the field $h$ on a randomly chosen site be the same at times $t$ and $\tau$. Quantity $m$ follows from Eq. (11) letting $m=x \varepsilon_{a} \varepsilon_{w} H / \sigma^{3}$, $c_{1}=4$, and $c_{2}=2 . \omega(t-\tau)$ depends on $t-\tau$ through the probability $\phi(t-\tau)$ that a spin point in opposite directions at times $t$ and $\tau$. To make progress, we make the approximation $\omega \simeq \min \left[2 \pi h_{0} \phi, 2 \sigma \sqrt{\pi} \sqrt{\phi}\right]$. The approximation for $\phi \ll 1$ follows from the Lorentzian PDF, of half width $h_{0} \phi$, that ensues when a small fraction $\phi$ of sites are randomly occupied by spins [16]. (The two factors of 2 come from the fact that flipping an already present spin $S$ is like placing a $2 S$ spin on an unoccupied site.) The approximation for $\phi \sim 1 / 2$ follows from the Gaussian distribution that holds then. In between, the interpolation checks, within some $10 \%$, with our MC results. Finally, $\phi(t-\tau)$ must be evaluated. For this purpose, an equation for the fraction of spins $n(t)$ that have flipped at least once before time $t$ is derived [15]. It is Eq. (11) using $n=x \varepsilon_{w} / \sigma, c_{1}=1$ and $c_{2}=1$. We then use $\phi \approx n / 2$. The functional dependence of $F$ shown in Eq. (2) follows by careful inspection of these equations. Numerical calculations yield the curves shown in Figs. 1(a), (b), and (c). The exponent $p$ depends on lattice structure, through $\sigma / h_{0}$ in the expression for $\omega(\phi)$ above. The agreement with our MC results exhibited in Fig. 1(c) is reassuring.

A couple of final remarks follow. Equations (2-5) are for magnetic systems that do not exchange energy with a heat bath (i.e., the lattice, usually) at very low temperatures. Then, $m(t) \rightarrow m_{e}$ as $t \rightarrow \infty$. When heat exchange does takes place readily, as in some systems in Ref. 13], then our simulations show that $m(t)$ eventually crosses over from the value given by Eqs. (2-5) to $m_{t h}(t) \simeq 0.3\left(\varepsilon_{w} / \sigma\right)^{3} H \Gamma t$, for $H \lesssim 1$. This happens when $m_{t h}(t)$ becomes the larger of the two. Later on, $m_{t h}$ levels off to a quasi steady state value (not the final thermal equilibrium value), which depends on both system shape and lattice structure, as expected. (If a field is not applied inmediately upon quenching, but later, the quasi steady state value of $m_{t h}$ is also affected.) The $\left(\varepsilon_{w} / \sigma\right)^{3}$ dependence suggests that (see Ref. 11]), in contrast with constant energy magnetization processes, magnetic ordering takes place while the system magnetizes.

Summing up, we have given MC and theoretically based evidence to show that the $m \propto \sqrt{t}$ behavior observed in experiments on $\mathrm{Fe}_{8}$ clusters [9] after quenching and applying a small field $H$ at $t=0$ is driven by correlations which are previously established in the system while cooling to very low temperature. We have established that the $\sqrt{t}$ behavior is not universal. More gener- ally, $m(t) \propto t^{p}$, and $p$ depends on lattice structure. The time range over which this behavior prevails, the value $m_{e}$ that the magnetization later levels off to, and the crossover time to a final thermally-driven regime have been determined. More specifically, Eq. (2-5) have been inferred from MC simulations and Eq. (11), and much of the relevant physics has been explained.

We are grateful to F. Luis and W. Wernsdorfer for useful comments. The Ministerio de Ciencia y Tecnología of Spain supported this work through grant No. BFM20000622.

* E-mail address: JFF@Pipe.Unizar.Es

[1] See, for instance, D. Ruizmolina, G. Christou, and D. N. Hendrickson, Mol. Cryst. Liq. Cryst. 343, 335 (2000).

[2] For $\mathrm{Fe}_{8}$, see W. Wernsdorfer and R. Sessoli, Science 284, 133 (1999); for $\mathrm{Mn}_{12}$, see F. Luis, J. Bartolomé, and J. F. Fernández, Phys. Rev. B 57, 505, (1998).

[3] C. Sangregorio, T. Ohm, C. Paulsen, R. Sessoli, and D. Gatteschi, Phys. Rev. Lett. 78, 4645 (1997); for tunneling through thermally excited states, see J. R. Friedman, M. P. Sarachik, J. Tejada, and R. Ziolo, Phys. Rev. Lett. 76, 3830 (1996); J. M. Hernaández, X. X. Zhang, F. Luis, J. Bartolomé, J. Tejada, and R. Ziolo, Europhys. Lett. 35, 301 (1996); L. Thomas, F. Lionti, R. Ballou, D. Gatteschi, R. Sessoli, and B. Barbara, Nature (London) 383, 145 (1996).

[4] N. V. Prokof'ev and P. C. E. Stamp, Phys. Rev. Lett. 80, 5794 (1998); Rep. Prog. Phys. 63, 669 (2000).

[5] D. A. Garanin, E. M. Chudnovsky, and R. Schilling, Phys. Rev. B 61, 12204 (2000); D. A. Garanin, E. M. Chudnovsky, Phys. Rev. B 65, 094423 (2002).

[6] T. Ohm, C. Sangregorio, and C. Paulsen, Eur. Phys. J. B 6, 195 (1998); L. Thomas and B. Barbara, J. Low Temp. Phys. 113, 1055 (1998); L. Thomas, A. Caneschi, B. Barbara, Phys. Rev. Lett. 83, 2398 (1999); W. Wernsdorfer, R. Sessoli, D. Gatteschi, Europhys. Lett. 47, 254 (1999).

[7] A. Cuccoli, A. Fort, A. Rettori, E. Adam, and J. Villain, Euro. Phys. J. B 12, 39 (1999).

[8] E. M. Chudnovsky, Phys. Rev. Lett. 84, 5676 (2000); N. V. Prokof'ev and P. C. E. Stamp, Phys. Rev. Lett. 84, 5677 (2000); W. Wernsdorfer, C. Paulsen, and R. Sessoli, Phys. Rev. Lett. 84, 5678 (2000).

[9] W. Wernsdorfer, T. Ohm, C. Sangregorio, R. Sessoli, D. Mailly, and C. Paulsen, Phys. Rev. Lett. 82, 3903 (1999).

[10] In numerical work, we use $\sigma \simeq 3.83,4.01$, and 8.42, which are the standard deviation values (slightly larger than the corresponding rms values) for $\mathrm{SC}, \mathrm{BCC}$, and $\mathrm{FCC}$ lattices, respectively.

[11] For why such an Ising model is appropriate for SMM's at very low temperatures if spin flip rules that include Eq. (1) are enforced, see J. F. Fernández, Phys. Rev. B 66 064423 (2002).

[12] L. Bokacheva, Andrew D. Kent, and M. A. Walters, Phys. Rev. Lett. 85, 4803 (2000).

[13] A. Morello, F. L. Mettes, F. Luis, J. F. Fernández, J. Krzystek, G. Aromí, G. Christou, and L. J. de Jongh, Phys. Rev. Lett. 90, 017206 (2003); M. Evangelisti, F. Luis, F. L. Mettes, N. Aliaga, G. Aromí, G. Christou, 
and L. J. de Jongh, Polyhedron (in press).

[14] J. J. Alonso and J. F. Fernández, Phys. Rev. Lett. 87, 097205 (2001).

[15] This can not be fitted here. It will be given elsewhere.
[16] A. Abragam, Principles of Nuclear Magnetism (Oxford Science Publications, Oxford, 1996) pp-125-128. 